\title{
Identifications totémiques : réflexions sur la relation entre humains et entités surnaturelles chez les Salish centraux de la côte
}

Totemic identifications: reflections on the relationship between humans and supernatural entities among the Central Coast Salish people Identificaciones totémicas: reflexiones sobre la relación entre humanos y entidades sobrenaturales en el grupo de los salish de la Costa central

Baptiste Gille

\section{OpenEdition}

Journals

Édition électronique

URL : https://journals.openedition.org/jsa/14319

DOI : 10.4000/jsa. 14319

ISSN : 1957-7842

\section{Éditeur}

Société des américanistes

\section{Édition imprimée}

Date de publication : 31 décembre 2015

Pagination : 105-136

ISSN : 0037-9174

Référence électronique

Baptiste Gille, « Identifications totémiques : réflexions sur la relation entre humains et entités surnaturelles chez les Salish centraux de la côte », Journal de la Société des américanistes [En ligne], 101-1 et 2 | 2015, mis en ligne le 15 mars 2016, consulté le 24 février 2023. URL : http:// journals.openedition.org/jsa/14319; DOI : https://doi.org/10.4000/jsa.14319 


\title{
Identifications totémiques : réflexions sur la relation entre humains et entités surnaturelles chez les Salish centraux de la côte
}

\author{
Baptiste GILLE *
}

\begin{abstract}
Actuellement, de nombreux autochtones vivant dans la région de Vancouver, sur les territoires des Salish centraux de la côte, affirment entretenir un rapport particulier avec une entité surnaturelle, souvent une espèce non humaine animale, végétale ou un élément du paysage. L'objectif de l'article est d'éclairer les différentes modalités de cette identification à un non-humain surnaturel protecteur, en partant des hésitations, embarras, imprécisions qui surviennent lorsque l'on cherche à la définir. Se dessinent alors les contours d'un véritable " complexe totémique », une structure flottante autorisant plusieurs relations possibles avec des non-humains. Cette étude propose donc de reconsidérer la question du totémisme chez les Salish côtiers et de montrer comment elle peut s'approfondir si le totémisme est appréhendé comme un « complexe » relationnel souple, consistant dans la coexistence de plusieurs combinaisons relationnelles possibles et cohérentes avec un non-humain surnaturel. [Mots-clés : Salish côtiers, totémisme, esprit protecteur, identification, incertitude.]
\end{abstract}

Totemic identifications: reflections on the relationship between humans and supernatural entities among the Central Coast Salish people. Nowadays, many indigenous people living in the Vancouver area, on Central Coast Salish territories, can claim to have a special relationship with a supernatural entity, often a non-human animal species, a vegetal species, or an element of the landscape. The purpose of this article is to highlight the different modalities of this identification with a protective non-human being, and it takes as its starting point hesitations, difficulties, and inaccuracies that occur when one tries to define such identification. By doing so, we outline a real « totemic complex », i.e. a floating structure allowing several possible relationships with a non-human. This paper therefore proposes to reconsider the question of totemism among the Coast Salish people, and to show how it can be deepened if totemism is apprehended as a relational and flexible « complex », involving several possible relational and consistent combinations with a supernatural being. [Key words: Coast Salish, Totemism, guardian spirit, identification, uncertainty.]

* Musée du quai Branly, département de la recherche et de l'enseignement, Paris [baptiste. gille@yahoo.fr].

Journal de la société des américanistes, 2015, 101-1 et 2, p. 105-136. C Société des américanistes. 
Identificaciones totémicas: reflexiones sobre la relación entre humanos y entidades sobrenaturales en el grupo de los salish de la Costa central. Actualmente numerosos habitantes autóctonos de la región de Vancouver, en los territorios salish de la costa, afirman poseer una relación particular con un ente sobrenatural, ya sea una especie animal o vegetal no humana o un elemento del paisaje. El presente artículo pretende esclarecer las diferentes modalidades bajo las cuales se produce esta identificación con una entidad no humana protectora, tomando en cuenta las dudas, dificultades e imprecisiones que se presentan a la hora de intentar definirla. Es entonces cuando se dibujan los contornos de un verdadero « complejo totémico », es decir de una estructura flotante a través de la cual pueda ser considerada la diversidad de relaciones posibles con los no-humanos. El estudio plantea una reconsideración del totemismo salish de la costa, mostrando cómo éste puede ser profundizado si el totemismo es aprehendido como un « complejo » relacional flexible que comprende la coexistencia de numerosas combinaciones relacionales posibles y coherentes con un sobrenatural no humano. [Palabras clave: Salish de la costa, totemismo, espíritu protector, identificación, incertidumbre.]

De nos jours, de nombreux autochtones Salish habitant le long de la côte pacifique, dans la région de Vancouver, continuent d'entretenir, en marge de leur insertion dans la vie socio-économique nord-américaine, un rapport particulier aux traditions de leurs ancêtres. Certains schèmes de rapport au monde, que l'on pourrait qualifier de « totémiques », continuent de subsister en parallèle, ou parfois contre, le mode de vie dominant de la société des « blancs », descendants des colons euro-américains. Ainsi beaucoup d'interlocuteurs peuvent affirmer qu'ils maintiennent un rapport privé avec une entité non humaine surnaturelle, souvent une espèce animale de la région. L'objectif du présent article est d'analyser les différentes modalités de cette relation à un non-humain surnaturel et les types d'identification qu'elles mobilisent.

Ce lien avec une entité surnaturelle est souvent présenté comme une relation intime, souffrant d'être trop explicitée. Lorsque l'on cherche à l'éclaircir plus avant, ce lien est exprimé avec précaution, à travers des hésitations et de profondes incertitudes. Ces hésitations laissent transparaître une forme d'indécision récurrente quant à la nature de l'entité surnaturelle ou à la relation adéquate à adopter avec elle. Pourtant, derrière ces hésitations, cette impression de flou, certains schèmes explicatifs sont continuellement convoqués. Le présent travail part de l'idée que loin d'être secondaires et périphériques, ces tâtonnements mêmes, ces embarras et incertitudes réitérées quant à la nature de l'entité surnaturelle, expriment au mieux le totémisme, c'est-à-dire la pluralité possible des rapports concevables entre un être humain et un être protecteur. Il s'agit donc de mettre en lumière ce qui pourrait être nommé un " complexe totémique », un groupe cohérent de relations possibles aux entités surnaturelles, actualisées différemment selon les individus. Cette nouvelle perspective permet de résoudre bien des problèmes dans le débat, plus que centenaire, sur le totémisme des Salish côtiers. 
Les individus hésitent dans leurs explications, mais ne restent pas pour autant silencieux ou sans justification. Il s'agit donc dans cette étude de prendre au sérieux l'ensemble des données concernant les entités surnaturelles - c'est-à-dire à la fois les hésitations (élément discursif négatif) et les explications (élément discursif positif). Ainsi, plusieurs combinaisons relationnelles cohérentes peuvent coexister, permettant le flottement de l'une à l'autre sans trop de contradiction. Tracer les contours de ce complexe totémique chez les Salish côtiers permettra de comprendre comment différentes motivations individuelles peuvent se rencontrer sans contradiction autour d'un noyau commun de pratiques.

\section{Modalités des énonciations sur l'expérience du surnaturel}

\section{Brouillages communicationnels et discours communs}

Je me trouvai, un soir, sur un territoire des Salish centraux de la côte, sur la réserve de Coldwater ${ }^{1}$, vers la ville de Merritt, au nord-est de Vancouver, et je devais rendre compte à un ancien (elder) qui me le demandait des intentions qui me poussaient à vouloir continuer ma participation aux rituels. Avec embarras, j'exprimai l'idée que j'étais peut-être seulement curieux de connaître la nature des entités surnaturelles auxquelles il faisait souvent référence, vers lesquelles les rituels devaient conduire. L'ancien laissa passer un temps de silence et sembla choisir ses mots. Puis il me répondit calmement que cette attitude n'était pas forcément saine, car le monde des forces et entités surnaturelles est particulièrement dangereux : «On ne peut vouloir seulement y aller par curiosité. C'est un monde terrifiant. Il faut y aller pour y chercher quelque chose. La route est longue pour s'y préparer. »

Ce qui me marqua d'abord dans les conseils de cet ancien, c'est le caractère solennel et grave qu'ils avaient revêtu. Si au quotidien j'avais déjà pu l'entendre parler avec humour et légèreté de sujets parfois profonds, j'avais aussi pu remarquer que tous ses comptes rendus sincères sur l'expérience du surnaturel dans le cadre d'une transmission de savoir étaient entourés d'une aura de solennité. D'une manière générale, lors du rappel d'une expérience surnaturelle, il est possible de remarquer chez de nombreux anciens des mécanismes de brouillage des marqueurs énonciatifs. Par exemple ici, les mots de l'ancien étaient renforcés par l'emploi de déictiques vides (« $C$ 'est », «s' $y$ préparer », « $y$ aller », « $y$ chercher », etc.) : le discours renvoyait à cette expérience du surnaturel, sans la spécifier ou la déterminer plus avant, formant autour d'elle un halo de

1. Ces analyses font suite à une expérience de terrain de quinze mois passés dans la région de Vancouver entre 2008 et 2009. Je tiens à remercier toutes les personnes qui ont bien voulu m'accepter et me guider dans la vie rituelle, partager du temps - de l'amitié - et accepter avec bienveillance mes questions parfois intempestives. 
mystère. Les procédés énonciatifs décrivant une expérience du sacré des Salish côtiers cherchent ainsi souvent à éviter les mécanismes de désambiguïsation de la communication ordinaire (Sperber et Wilson 1989), c'est-à-dire ceux qui obligent le locuteur, dans des conditions normales de communication, à se rendre pertinent et à transmettre un maximum d'information avec un minimum d'effort pour l'interlocuteur. Ces procédés créent ainsi une structure d'attente chez l'interlocuteur et dessinent les contours de l'expérience surnaturelle comme ceux d'un objet indéterminé (Gille 2014).

Cela n'est pas nouveau. Alors que les entités surnaturelles ont toujours occupé une grande place dans l'organisation de la vie sociale, les premières études ethnographiques rappellent que les Salish ont toujours été peu loquaces à leur sujet, notamment parce qu'elles étaient soumises à des droits de propriété et à divers tabous (Hill-Tout 1904 ; Wike 1941 ; Suttles et Jenness 1956). Aujourd'hui il est encore dit que parler d'une entité surnaturelle, c'est convoquer son pouvoir, ce dernier pouvant se faire voler ou diminuer à force d'être mobilisé sans raison. Certaines de ces justifications de prohibition sont résumées dans les propos du représentant culturel Stó: $1 \bar{o}^{2}$ Nawawalhts 'i, Albert Sonny McHalsie, lors d'un entretien avec l'anthropologue Bruce Granville Miller :

Si quelque chose de spécial nous arrive, nous n'avons pas le droit d'en parler. Comme par exemple une rencontre avec le sasquatch. C'est pour cela que beaucoup d'autochtones ne partagent pas leurs histoires de rencontre avec le sasquatch parce qu'on nous apprend que c'est quelque chose de spécial, et que ce n'est pas quelque chose dont on parle. Si tu en parles trop, cela perd de son pouvoir. (Miller 2007, p. 124 ; traduction de l'auteur ${ }^{3}$ )

Malgré une référence explicite à la rencontre avec le sasquatch - une sorte de big foot - on peut ici de nouveau relever la construction énonciative d'une expérience surnaturelle sans référence déterminée, par l'emploi de déictiques vides (" quelque chose de spécial », " quelque chose », " en parler », " cela perd de son pouvoir », etc.).

Si les anciens restent souvent évasifs, une manière courante d'enrayer les questions sur les esprits consiste, à l'inverse, à les noyer dans des discours communs et des généralités. Lors de discussions informelles, avant ou après des cérémonies de sudation - c'est-à-dire des cérémonies de chants et prières qui se déroulent à l'intérieur de tentes ou étuves chauffées -, des participants pouvaient me donner certaines informations plus précises sur les entités surnaturelles, tout en restant pourtant aussi vagues que possible sur la relation intime qu'ils pouvaient entretenir avec elles. Généralement, dans ces cas, la

2. Le groupe Stó:lō est une communauté Salish de la côte vivant à l'est de Vancouver.

3. Toutes les traductions sont de Baptiste Gille, elles apparaîtront suivies de la mention « traduction de l'auteur». 
description du lien avec une entité surnaturelle était encadrée d'un mélange de vulgate new age, de principes de psychologie positive et de préceptes de bien-être. Ces récits permettent le plus souvent de créer une première forme de consensus, constituant notamment des lieux de recoupement avec l'imaginaire euro-américain, que tout le monde partage.

Il est possible de saisir ce type de syncrétisme new age à travers trois courts exemples :

1. Une femme d'une quarantaine d'années, qui participait à des cérémonies de sudation et à qui je posai la question de savoir pourquoi les rituels de sudation étaient dits améliorer le contact avec le monde surnaturel, m'expliqua que c'était parce que les chants purifiaient l'âme et les plantes médicinales nettoyaient le corps, comme lors d'un jeûne, mais aussi comme lorsque l'on pratique le yoga ou la méditation. Si la conception d'une purification du corps est ancienne - de nombreux récits dans la littérature ethnographique sur les Salish côtiers font état de la nécessité de se laver régulièrement le corps et de se brosser avec des branches de cèdre lors des quêtes de vision (Suttles et Jenness 1956, p. 65 et suiv.) -, la référence au yoga fait partie d'un nouveau type de syncrétisme cherchant à rapprocher les pratiques autochtones des pratiques de purification orientales, comme le bouddhisme et les techniques du corps indiennes.

2. J'ai pu participer à des ateliers de travail à l'université de ColombieBritannique dans lesquels la spiritualité autochtone salish et plus généralement le statut pan-autochtone d'ancien étaient présentés comme un modèle de leadership. Le statut d'ancien pouvait alors servir à affirmer les qualités de leader, le rayonnement personnel, voire la réussite entrepreneuriale. On parlait ainsi sans tabou de la sagesse et de l'expérience du surnaturel acquises par les anciens, mais dans des idiomes éloignés des cadres culturels qui les avaient vus naître.

3. Enfin, lors d'une journée de conférence sur la médecine autochtone, toujours à l'université de Colombie-Britannique, j'ai pu m'entretenir avec une auditrice autochtone. Elle me fit part du fait que selon elle les pratiques thérapeutiques autochtones pouvaient être pensées comme des manipulations d'énergie. Il était possible de concevoir la religion autochtone à partir de l'idée empruntée au vocabulaire de la science moderne de la matière comme énergie : les entités surnaturelles étaient peut-être des sortes de «pôles » concentrés d'énergie. Là encore, la nature des entités surnaturelles était comprise à l'intérieur d'une interprétation new age de la notion d'énergie et les modalités énonciatives étaient emboîtées dans ce contexte qui leur faisait perdre tout caractère tabou.

Il faut donc peut-être différencier dans un premier temps, sans pour autant en faire une délimitation trop rigide, le « savoir institué » de la communauté des anciens du « savoir commun » ${ }^{4}$, c'est-à-dire des justifications qui se transmettent

4. Cette distinction entre savoir institué et savoir commun est empruntée à Pierre Déléage dans son étude sur le chamanisme des Sharanahua (Déléage 2009). 
de façon plus relâchée. Les anciens, détenteurs d'un savoir plus fondé, parce que supposés avoir une expérience du surnaturel plus étendue, modifient avec prudence les marqueurs énonciatifs, laissant planer davantage de mystère. Au contraire, quand je m'entretenais, dans les premiers temps sur un mode informel, avec des participants aux cérémonies de sudation - détenteurs d'un savoir plus commun -, on me donnait généralement des justifications assez courantes, constituées de références spirituelles éclectiques puisées dans la littérature new age.

\section{Une déférence épistémique à l'égard du savoir des anciens}

Si peu de gens entrent dans le détail, quand il est question de la relation « privée » qu'ils entretiennent avec une entité surnaturelle, la possibilité d'une expérience ostensive, directe, à travers des rêves ou des coïncidences étranges, est souvent considérée comme la marque du savoir des anciens. Les personnes possédant un savoir ordinaire sur ces questions renvoient souvent par « déférence » au savoir des anciens. Par déférence, il faut entendre ici une forme de respect pour la parole ou la connaissance d'une personne que l'on reconnaît comme ayant une meilleure expérience ou connaissance que soi-même sur certains sujets ${ }^{5}$.

Lorsque je demandai à un ami pourquoi il avait décidé de planter sur la colline deux couteaux au sud d'un cercle sacré dans lequel il s'apprêtait à faire une prière, il me répondit simplement qu'un ancien lui avait dit de faire ainsi, sans autre justification. Autre exemple de déférence simple : lorsque je cherchais à connaître davantage la nature de l'entité surnaturelle que l'on nomme oiseau-tonnerre (thunderbird en anglais ou shxwexwo:s en halkomelem, langue autochtone qui n'est utilisée que dans de rares cas, notamment en référence à des termes sacrés), je fus renvoyé à plusieurs reprises vers des anciens censés mieux connaître cette entité particulière pour l'avoir eux-mêmes rencontrée en vision. On défère envers certaines personnes en s'appuyant sur l'étiquette implicite d' « ancien » : il s'agit de toute personne reconnue par la communauté comme détentrice d'un savoir concernant les pratiques culturelles autochtones, souvent en accord avec les traditions locales ou du moins avec des préceptes pan-autochtones plus généraux.

$\mathrm{Au}$ bout de la chaîne de déférence, comment se donne donc le savoir des anciens ? Comme nous l'avons vu, le savoir des anciens concernant les entités surnaturelles ne se présente jamais sous une forme explicite ou descriptive. $\mathrm{Au}$ contraire il mobilise constamment des marqueurs énonciatifs aux effets d'ellipses, ainsi que des références équivoques, et s'appuie sur des modifications

5. L'utilisation faite ici du concept de déférence est largement inspirée de celle faite par Bloch (2005) et Déléage (2009). 
des règles communicationnelles. Ces modifications sont-elles dès lors le signe qu'il n'existe pas de connaissance sur la nature des entités surnaturelles et les types de relations?

Les anciens laissent en réalité transparaître leur connaissance des entités surnaturelles et des relations à entretenir avec elles de deux manières : 1. beaucoup de comptes rendus d'expériences avec les entités surnaturelles se donnent à la marge, dans des discours informels, comme en passant; 2. le manque de loquacité marque la volonté de rappeler que ces expériences ne sont véritablement appréhendables qu'en première personne. Ces processus de brouillage propres aux énonciations des anciens sur ces questions semblent plutôt destinés à constituer des renvois directs et ostensifs de l'interlocuteur aux mêmes types d'expériences. Il s'agit de rappeler à celui intéressé par ces questions que le rapport authentique aux entités surnaturelles ne peut pas être qu'un rapport de déférence, de discours rapportés ou même de description : il faut toujours pouvoir expérimenter cette relation par soi-même et cela à travers un engagement solide dans la préparation rituelle, dont sont en charge ces mêmes anciens. Ce n'est que lorsque l'individu fera preuve d'un grand investissement et d'une participation constante aux rituels que certains témoignages pourront être partagés de manière informelle.

Un jour que nous faisions partir le feu pour chauffer les pierres pour une cérémonie de sudation, je demandai à un ancien, responsable de la loge, s’il pensait que les « blancs » et les autochtones pouvaient rencontrer les mêmes entités surnaturelles ou si elles étaient spécifiques à chaque culture. Il me répondit qu'il pensait que les entités étaient identiques, même si elles pouvaient apparaître différemment à ceux qui ne les connaissaient pas. Selon lui, les rituels permettaient d'approcher les entités surnaturelles directement et ces entités étaient les mêmes que celles de leurs ancêtres. On peut remarquer, alors que ma question concernait la nature des entités surnaturelles, que sa réponse me renvoya plutôt à la forme rituelle elle-même : c'est là un procédé ordinaire d'embrayage du discours des anciens sur le surnaturel qui, plutôt que de faire valoir des descriptions des entités, renvoient l'interlocuteur aux outils - en l'occurrence ici rituels - permettant de les expérimenter en première personne. À une autre occasion, ce même ancien me dit que les amérindiens ne devaient plus avoir honte de leurs rituels et de leurs croyances, qu'ils devaient les rendre accessibles à tous, notamment aux «blancs », pour leur montrer la force des esprits et pour que tous puissent voir que les «manières de faire » (the way we do) autochtones sont puissantes (powerful).

Ainsi, d'une manière générale, les anciens donnent rarement des leçons détaillées sur la nature des entités surnaturelles et font peu état de cartographies cosmologiques complexes. Ils se réfèrent à leur « manière de faire " (the way we do), plutôt qu'à leur manière de penser. Ils attendent davantage des individus 
intéressés par ces questions qu'ils expérimentent d'eux-mêmes les réponses à leurs questions, à travers la participation aux activités rituelles.

Il faut donc partir du double constat que les discours au sujet des entités surnaturelles sont particulièrement tâtonnants, empreints de fortes hésitations, précautions et avertissements, mais ne sont pas non plus totalement prohibés ou fermés. Ces flottements justificatifs ne sont pas nouveaux chez les Salish côtiers. Jenness remarquait déjà, au début du siècle dernier, que, concernant la nature de l'entité protectrice surnaturelle et de l'âme, les anciens des communautés Saanisch de l'ouest n'étaient pas d'accord avec les Nanaimo de l'île et les Sardis des Basses Terres, et n'étaient en outre pas d'accord entre eux (Suttles et Jenness 1956, p. 89). Cette grande disparité théorique est aussi remarquée par Hultkrantz au milieu du $\mathrm{xx}^{\mathrm{e}}$ siècle : «Le matériel-source concernant les conceptions de l'âme parmi les nombreuses tribus Salish de la côte et de l'intérieur, et spécialement parmi ces derniers, est très inégal » (Hultkrantz 1953, p. 65 ; traduction de l'auteur). Les anciens Salish centraux de la côte, interrogés par Wells dans les années 1960, rappelaient aussi souvent que leurs propres anciens connaissaient mieux ces questions sur les entités surnaturelles qu'euxmêmes et qu'ils ne pouvaient donc pas s'étendre beaucoup sur ces questions (Wells 1987, p. 159).

Les comptes rendus de rencontres avec des entités surnaturelles ne se font donc pas via des récits, comme cela peut être le cas dans d'autres régions du monde. L'ethnographie que je propose repose donc principalement sur des témoignages faits « en passant ». Si certaines confidences transparaissent aujourd'hui, elles sont souvent faites à la marge, lors de discussions détendues, comme des parenthèses discrètes à l'intérieur d'autres conversations. C'est sur ces témoignages informels, ainsi que sur les justifications de la pratique rituelle, que nous nous appuyons maintenant pour comprendre la diversité des liens entretenus aujourd'hui avec les entités surnaturelles.

\section{Forme des identités totémiques}

\section{Une relation vague de parenté}

Malgré le peu de loquacité des Salish de la côte, certaines conceptions au sujet de l'entité protectrice reviennent de manière récurrente. Même à travers des explications flottantes, les individus hésitent dans le cadre restreint d'un certain nombre de schèmes et de possibilités de justifications. Ce sont ces oscillations singulières, ces indécisions et ces incertitudes récurrentes qui permettent d'exprimer une pluralité cohérente de rapports aux entités surnaturelles et qui forment ce que l'on pourrait nommer un « complexe totémique ». Le totémisme, que nous définissons pour l'instant avec Frazer (1887, p. 3 ; Rosa 2003, p. 232) 
comme la relation d'un individu à une entité surnaturelle non humaine, la plupart du temps protectrice, apparaît d'abord à travers l'identification d'une structure vague et incertaine de parenté.

Un jour que je participais à un rituel de deuil, nous vîmes tous plusieurs aigles voler au-dessus de l'endroit où nous nous trouvions. Étonné par un aussi grand rassemblement, je levai la tête et montrai du doigt les aigles à un ancien dont j'étais proche. J'appris alors qu'on ne pouvait pas montrer les aigles du doigt car c'est toujours manquer de respect que de désigner de cette manière ses grands-parents. Il était question ici de mes propres grands-parents. Pour les anciens présents, les aigles apparaissaient un peu comme les grands-parents de tous ceux qui étaient réunis ici. Nous faisions alors partie d'une loge de pratiques rituelles mise sous le signe de l'aigle et de la compassion, mais je n'avais jamais fait part d'une relation particulière avec les aigles. En réalité, il semblait peu importer que ce soit l'aigle ou un autre animal, et la relation semblait tout autant pouvoir concerner une autre personne que moi.

Cette expérience rappelle la présence d'une identification totémique a minima très relâchée et flottante. Ainsi un Français blanc, novice dans la pratique rituelle, devrait pouvoir reconnaître les aigles comme ses ancêtres et, surtout, entrer avec eux sous le même schème de respect et de politesse que celui valorisé chez les Salish de la côte entre petits-enfants et grands-parents (sur cette relation privilégiée entre petits-enfants et grands-parents chez les Salish côtiers, voir entre autres Barnett 1955, p. 132-135; Amoss 1978, p. 17 ; Carlson et McHalsie 2001, p. 29). Le rapport aux non-humains en tant qu' « esprits » (la nature de ce terme sera précisée plus loin) est donc d'abord celui d'une parenté indéterminée, dont la modalité est celle d'une protection réciproque (l'humain et le non-humain surnaturel se protègent réciproquement, comme les grands-parents et les parents protègent les enfants). Le schème vague de parenté permet de faire état au mieux de cette relation (sous la forme énonciative, par exemple, « Je suis relié à l'ours », « Je suis connecté à tel rocher», etc.). Cette relation de parenté vague peut-être exprimée soit sous la forme d'une relation d'alliance indéterminée, soit sous celle d'une filiation indéterminée (il est dit que les aigles sont les grands-parents de tout le monde).

Cette relation de parenté vague peut d'abord être exprimée sous la forme d'une alliance indéterminée qui se présente sous l'aspect d'une résonance singulière. Le terme « résonance » n'est pas issu de l'usage vernaculaire, mais il permet de rendre compte d'une affinité de longue date, parfois justifiée par l'évocation de souvenirs d'enfance. Un participant actif à la vie rituelle, aux origines salish de la côte et mi'kmaq, qui avait souvent prié le bison lors de rituels de sudation, me fit part lors d'une discussion sur cette question du fait que son entité protectrice était d'abord venue en rêve de manière récurrente, puis en quête de vision, et qu'il avait toujours senti la force de cet animal en lui depuis sa petite enfance : 
«Lorsque je vois un bison, je peux sentir en lui des choses. On se comprend, on sent et on pense de la même manière. " Cette identification avec le bison ne semblait pas anodine : cette personne était singulièrement corpulente et il est possible de penser qu'elle avait pu développer un ressenti à la mesure de sa propre force, sous le rapport d'un certain nombre de saillances physiques. Cette affinité est souvent comparable à l'expression d'une « tonalité » ou d'une « résonance » entre les comportements et la manière de penser d'un humain et ceux propres à tous les individus d'une espèce animale. Cette relation d'accordance presque musicale n'est pas anodine chez les Salish centraux de la côte puisque souvent l'individu possède des chants propres, chargés de pouvoir, offerts par l'entité surnaturelle et qui permettent de manifester sa présence (Amoss 1978 ; Jilek 1974 ; Kew 1970). Il existe donc une manière de penser cette « alliance » singulière, intime et individuelle avec une entité surnaturelle sur le modèle d'une résonnance. Par « alliance », il faut ainsi comprendre un certain lien d'apprivoisement qui se construit avec le temps et qui repose sur l'identification selon des principes d'accordance physique ou mentale.

Si dans le rapport au bison décrit plus haut, il est davantage question d'une affinité personnelle, individuelle, du corps et de l'esprit avec l'animal, ayant le caractère d'une alliance, dans d'autres cas, le lien relève plutôt d'une affinité familiale, justifiant encore davantage la mobilisation d'une relation de parenté. Certaines entités sont héritées par lignage cognatique. Par exemple, sur le territoire musqueam, au cœur de Vancouver, plusieurs anciens peuvent encore aujourd'hui faire état d'un lien familial ancestral avec l'oiseau-tonnerre. Ce lien se manifeste parfois par des dimensions affectives fortes. Un jour, lors d'une cérémonie de passation de pouvoirs, je devais exprimer au tambour, avec d'autres, la présence de l'oiseau-tonnerre, notamment le battement de ses ailes sous la forme de coups de percussion. Un ancien respecté vint alors nous dire que nous devions imaginer l'émerveillement que ressent toute personne qui voit venir vers lui l'oiseau-tonnerre. Il tentait de faire partager émotionnellement une vision et de rappeler le mélange de peur et d'émerveillement que nous devions rendre manifeste. Il avait pris le ton sérieux qui caractérise le partage d'une expérience des entités surnaturelles. Il nous fit part du fait que l'oiseautonnerre était une entité avec laquelle plusieurs membres de sa famille, dont lui-même, avaient tissé un lien. Une relation de parenté similaire, c'est-à-dire indéterminée, avec l'esturgeon a également été rapportée récemment dans la littérature par le représentant stó:lō Nawawalhts 'i, (Miller 2007, p. 105).

C'est en premier lieu une relation de parenté vague - entre « l'alliance » personnelle et la « filiation »-qui permet d'identifier le rapport à une entité surnaturelle. Turner (2005) remarque ainsi que la plupart des Salish font référence à une relation indéterminée, mais « centrée-sur-la-parenté » (kincentrism). De nos jours, cette notion vague de parenté est héritée de conceptions pan-autochtones 
plus générales, comme les notions de « relation 》 (relation) ou de « parents » (relatives), empruntées aux cultures des Sioux Lakota, aujourd'hui diffuses sur tout le continent nord-américain. L'expression lakota "Mitakuye Oyasin » (signifiant « Tout est relié »), qu'un grand nombre de personnes emploie parmi les Salish côtiers, dénote ce lien implicite qui relie les êtres, que beaucoup d'autochtones reconnaissent comme primordial ${ }^{6}$.

Au-delà de cette référence lakota, la parenté flottante, entre filiation et alliance, utilisée pour dénoter la relation aux entités surnaturelles, n'est pas nouvelle chez les Salish côtiers. Les anthropologues ont en effet remarqué depuis longtemps chez les Salish centraux de la côte une hésitation entre une forme collective et une forme individuelle de totémisme (Hill-Tout 1904) : dans le «totémisme collectif », certains individus appartenant à de hautes familles reçoivent en héritage des entités surnaturelles (filiation). Le « totémisme individuel», au contraire, dénote le fait que tous les individus doivent, très tôt, se mettre en quête d'entités surnaturelles pour s'en faire des alliés personnels et il arrive que ces entités expriment une individualité qu'ils ne partagent pas avec d'autres membres de leur famille (alliance). Hill-Tout remarquait ainsi que les entités surnaturelles partagées collectivement étaient nommées sululia et que celles qui étaient personnelles étaient nommées sulia. La forme nominale du verbe ulia est la même dans les deux cas et signifie « rêver » ${ }^{7}$.

Il est possible que cette forme relationnelle particulièrement souple résulte de plusieurs facteurs : la destruction des formes communautaires autochtones avec l'arrivée des colons, l'introduction de logiques cognatiques occidentales, l'introduction du christianisme et de ses configurations singulières de parenté (tous les êtres sont des frères et sœurs, enfants d'un dieu unique), mais aussi les nouvelles manières pan-autochtones de construire le lien entre les existants, inspirées des cosmologies lakota. Ces nouvelles façons de penser la relation aux entités surnaturelles sont ainsi venues s'ajouter aux rapports originellement hésitants chez les Salish côtiers entre l'alliance et la filiation, c'est-à-dire des formes de totémisme individuel (sulia) et de totémisme collectif (sululia).

6. D'une manière générale, les Salish côtiers que j'ai fréquentés étaient impliqués dans beaucoup de rituels d'origine sioux, notamment ceux qui entourent les Danses du soleil. Se référant toujours à une conception pan-autochtone du monde, ils me confessaient que pour eux il n'y avait pas de différence entre la spiritualité sioux et celle des Salish. Par exemple, les anciens étaient autant impliqués, et avec la même ferveur, dans l'organisation des Danses du soleil l'été - rituel sioux - que dans celle des Danses spirituelles l'hiver - rituel des Salish côtiers.

7. Aujourd'hui les entités sont désignées par un seul et même terme halkomelem : syuwél ( « entité protectrice »), reposant toujours sur l'idée de rêve et de chant protecteur (Paliye). Pour une analyse grammaticale de ce terme, voir Galloway 1994, p. 584. 


\section{L'entité surnaturelle comme propriété extérieure du soi}

Cette pluralité de conceptions hésitantes oscillant entre l'alliance et la parenté se manifeste à d'autres niveaux, notamment lorsqu'il s'agit de savoir si l'entité est une partie de soi («propre »-de l'ordre de l'être) ou si elle est extérieure à soi (« propriété »-de l'ordre de l'avoir). Là encore l'incertitude est de mise : les participants aux rituels de sudation font souvent référence aux entités surnaturelles sur le modèle de ce qui est possédé ou de ce qui est reçu (" what I have »). Ce rapport de possession n'est pas toujours évident et sa compréhension est soumise à des séries d'hésitations. Nous allons analyser trois différentes modalités de cette relation de possession : d'abord, paradoxalement, lorsque cette relation de possession est inexistante, ou du moins ne se présente pas comme une appropriation de l'humain et du non-humain surnaturel (l'entité offre, par exemple, un don sans qu'il n'y ait d'appropriation), puis lorsque cette relation de possession se manifeste implicitement à travers une forme de domination de l'humain (l'humain est alors protégé), et enfin une domination du non-humain surnaturel (l'humain est alors agressé et peut tomber malade).

Il existe d'abord certaines justifications de la rencontre entre un humain et une entité surnaturelle qui ne supposent pas de logique d'appropriation : il est seulement dit que l'humain se fortifie au contact de l'entité non humaine. Lors de ma participation à des rituels de quête de vision dans la région de Merritt, j'ai remarqué que beaucoup de conseils donnés au quêteur étaient centrés sur la force acquise lors de la confrontation avec une entité surnaturelle. Alors que je m'apprêtais à monter sur la colline pour jeûner quatre jours, un participant ayant une longue expérience des activités rituelles, qui pratiquait le jeûne depuis plusieurs années, me dit : «Tu ne recevras que ce que tu donneras. L'important, c'est d'avoir le courage d'y aller, d'offrir quelque chose et de recevoir quelque chose. \ Puis il me conseilla de faire attention à tous les sons, d'écouter les messages de tous les animaux qui passeraient à côté, même les plus petits. Leur seule présence même devait être interprétée comme un message. Nombreux sont les témoignages dans lesquels certaines personnes ont pu voir des ours passer ou même des pumas (cougars), mais ces derniers ne s'aventurent jamais près du cercle dans lequel se tient l'initié, comme si ces animaux savaient instinctivement qu'ils ne devaient pas briser le cercle rituel. On accentue ainsi le fait que les quêtes de vision sont là pour fortifier le courage. Ce même initié m'avait dit durant la préparation rituelle : « Si ces rituels ne te font pas peur, cela est mauvais signe. Sans la peur, il n'y a pas de bravoure. » Les rencontres avec les entités surnaturelles, nécessitant de passer quatre jours sans eau ni nourriture, ont donc, selon certaines justifications, d'abord pour but de fortifier la volonté et de révéler les individus courageux, car les entités surnaturelles peuvent être hostiles ou vouloir tester la volonté de l'initié. Dans ce cas, la rencontre avec l'entité surnaturelle est moins encadrée 
par une relation d'appropriation que par la possibilité d'une consolidation de son pouvoir personnel au contact d'un être plus puissant que soi. Cette conception a aussi été avancée récemment par un conseil d'anciens stó:1ō qui, réunis sur la question de savoir ce qui se transmettait de l'entité surnaturelle à l'humain, répondirent que l'individu gagnait et augmentait sa force de vie, son pouvoir personnel, moins de « l'appropriation » que de la «coexistence » répétée avec le non-humain surnaturel (voir le témoignage de McHalsie dans Miller 2007, p. 127).

Soit la seule coexistence avec l'entité permet à l'individu d'affermir son pouvoir personnel, soit il peut aussi être dit que l'être surnaturel « offre » un don ou un pouvoir lors de la première rencontre, si le cœur de celui qui le quête est suffisamment pur et clair. Avant de monter sur la colline pour la quête de vision, l'ancien qui coordonnait le déroulement rituel me dit que pour rencontrer ces entités il fallait que je garde la conscience bien claire ("keep focused »), de bonnes intentions (" with good intentions ») et que tout vienne du cœur ("from the heart ») grâce aux prières et aux fumigations de sauge et tabac. Cette idée selon laquelle les entités surnaturelles ne viennent voir que les personnes qui sont « propres » et « purifiées » (cleansed) - physiquement et spirituellement peut se retrouver dans la littérature ethnographique concernant les Salish côtiers (Suttles et Jenness 1956 ; Jilek 1974 ; Amoss 1978). Ce qui est ici souligné est que l'entité vient offrir un don (une guérison, une vision, un chant, la certitude d'une vocation, la réponse à des questions, etc.) à une personne qui a le cœur et les intentions suffisamment purs. Dans ce cas, il n'est toujours pas question d'《 appropriation » : l'entité vient seulement offrir un pouvoir particulier à celui qui a montré qu'il voulait vraiment de l'aide, par la peine qu'il était susceptible de s'infliger (un jeûne de quatre jours sans nourriture ni eau).

Enfin, dans la plupart des cas les justifications des individus mettent l'accent sur une relation de protection conçue sur le modèle d'un lent apprivoisement puis d'une appropriation : on revient des quêtes de vision avec une nouvelle entité surnaturelle, sous forme de possession ("what I got»). Cette possession justifie la relation de protection : elle peut être pensée comme un échange de bons procédés, à travers lequel des prières sont exaucées en échange de nourriture brûlée, de rituels de purification, d'aide aux autres, de bonnes actions accomplies, etc. L'entité surnaturelle ne veut que le bien de son protégé. Cette relation de protection peut également être perçue comme une forme de domination où l'entité est soumise et domptée à force de confrontation. Les justifications à ce propos sont assez floues.

Dans le cas d'une relation de protection bienveillante, il est souvent dit que les entités surnaturelles sont attirées par les individus qui accomplissent de bonnes actions. Ainsi, lorsque 1'on me proposa d'être « gardien du feu » (firekeeper) pour certaines cérémonies de la loge, la justification principale était que c'était 
une bonne action et que d'une manière générale la participation et l'aide aux activités rituelles étaient une des meilleures manières de travailler ses bonnes intentions. C'est une forme de sacrifice de soi qui rend meilleur. Il est admis que les comportements moralement bons purifient et la purification permet de trouver grâce auprès d'une entité surnaturelle. Dans ce cas, la relation à l'esprit est celle d'un apprivoisement doux, d'une alliance avec un ami intangible, sur le modèle d'un ange gardien. L'individu n'entre pas en conflit avec l'entité, elle est seulement à son service, protecteur bienveillant, dont la bonté est au-delà de toute lutte possible entre egos. Si cette conception est partagée par beaucoup d'individus, certains anciens pensent que les entités protectrices peuvent par contre être vues comme des puissances hostiles.

Les entités surnaturelles peuvent ainsi être présentées par les anciens comme prédatrices ou inamicales dans un premier temps. Les anciens donnent de nombreux avertissements aux novices; plusieurs gestes rituels sont destinés à ne pas offenser les entités surnaturelles. Par exemple, lorsque nous coupions du bois pour les cérémonies de sudation, nous ne devions jamais oublier de faire une prière propitiatoire et de poser une pincée de tabac sur le sol - quelques fois seulement avant de s'enfoncer dans la forêt mais parfois aussi pour chaque arbre que nous arrachions. Les entités surnaturelles s'offusquent facilement : il ne fallait pas cracher sur le sol pour ne pas manquer de respect aux esprits des lieux. Les esprits peuvent s'offenser de la désinvolture des humains, en particulier s'ils sont conspués : un individu qui refuse de voir le lien qui le lie à un esprit, qui fait par exemple du mal à certains animaux avec lesquels il est relié sans le savoir, peut tomber gravement malade. La langue halkomelem possède le terme xó:li:s (Carlson et McHalsie 2001, p. 8 ; Miller 2007, p. 128) qui se traduit par « se tordre et mourir ». Ce terme désignait autrefois une maladie étrange qui pouvait résulter d'une rencontre inopinée avec une entité surnaturelle. La maladie était pensée comme un « entortillement » (twist around) (voir les témoignages des anciens dans Wells 1987).

Les entités surnaturelles peuvent donc être rencontrées sur le mode d'une relation d'hostilité. Elles ont le pouvoir d'entraîner un individu dans les spirales de l'alcool ou de l'addiction, de le mettre en péril, qu'il les recherche ou pas. Dans ces cas, il faut de la force personnelle (spiritual power) pour les apprivoiser. Plus l'entité surnaturelle est initialement prédatrice, plus son pouvoir surnaturel sera efficace lorsqu'elle sera apprivoisée. À cet égard, certaines entités n'étaient autrefois recherchées que par des apprentis-guérisseurs ou chamanes qui, d'une part, étaient suffisamment forts pour les affronter et qui, d'autre part, avaient besoin de leur pouvoir pour les rites de guérison. Des entités hautement individualisées (qui n'existent qu'en un seul exemplaire) comme l'oiseau-tonnerre (shxwexwo:s), le serpent-à-deux-têtes (si:lhqey), les yeux-flamboyants (st'qoya), l'asticot (appel), l'ours-noir-vivant-sous-les-eaux 
(slalakum) ou encore le sasquatch (sésq̉ac), sont réputées vivre dans les forêts à l'est de Vancouver. Si l'on souhaitait acquérir le pouvoir personnel de ces entités, il fallait respecter un certain nombre de protocoles : reculer lentement les yeux fixés sur la créature jusqu'à ce que celle-ci soit hors de vue, s'arracher un cheveu et l'envoyer en soufflant vers la divinité. Si la créature sacrée ne se considérait pas offensée, elle pouvait offrir son pouvoir. Dans d'autres cas, il est dit que c'est moins un don de la créature qui est fait, qu'une sorte de rapt qui s'opère au détriment de l'entité : il s'agit vraiment de convaincre l'entité de s'offrir en sacrifice pour donner son pouvoir à l'individu ${ }^{8}$. Ces conceptions ont encore cours. Dans la ville de Hope, au sud de Merritt, je discutais un jour avec un homme qui participait à beaucoup d'activités rituelles et qui me dit que certains individus continuaient à chercher le sasquatch dans la région pour son pouvoir. Plusieurs participants aux cérémonies de sudation continuent de prendre au sérieux ces entités surnaturelles uniques, même si elles ont déserté les lieux, tandis que d'autres pensent que ce sont de vieilles croyances.

L'entité surnaturelle est ici conçue comme « une partie extérieure du soi ». Elle est ainsi soumise, encore aujourd'hui, à des protocoles d'acquisition : appropriation d'un pouvoir sur le modèle d'un don, d'un rapt ou d'un acte de prédation, ou encore à travers un apprivoisement (comme peut l'être celui d'un animal ou d'un ami). Dans ce dernier cas, la relation d'apprivoisement se fait en douceur ou résulte d'une lutte de domination, débouchant sur une relation asymétrique dominant-dominé. Dans les travaux ethnographiques du siècle passé, les esprits surnaturels pouvaient être «domptés » comme des chiens, voire des esclaves 9 . Lors des danses spirituelles d'hiver des Salish côtiers, durant lesquelles un initié en transe tente de dompter une entité surnaturelle qui le possède, il était souvent dit que l'individu ne devait jamais se laisser emporter par l'entité, mais toujours pouvoir la dominer et coopérer avec elle (Amoss 1978, p. 120). En général, aujourd'hui, la plupart des participants aux

8. La littérature ethnographique concernant les Salish est abondante sur cette question. Voir Carlson et McHalsie 2001, p. 8 ; Hill-Tout 1978, vol. 2, p. 73-76 ; Van Ejik 2001, p. 179 et 183 ; Suttles 1987, p. 75-76; Wells 1987, p. 54-55, 84-85 et 156-157.

9. Deux exemples. Concernant les Skagit septentrionaux, Collins rapporte le commentaire suivant : «C'est comme avec Sandy, le chien que je possède. Si je meurs, le chien ira certainement à un de mes enfants. Si aucun de mes enfants ne peut prendre soin de lui, il repartira vers le lieu d'où il vient, quel qu'il soit. Les esprits reviennent vers le lieu d'où ils viennent, quel qu'il soit. Si mes enfants ne peuvent pas les comprendre, ils seront offerts à la possession de n'importe qui » (1975, p. 181). Au sujet des Twana, Elmendorf rapporte ceci : "Quand une personne meurt son esprit ne va pas avec lui vers le pays des morts. Il l'a toujours suivi comme un chien, et quand il meurt, il est comme un chien perdu. Parfois, le pouvoir oublie son propriétaire mort, mais quelquefois il veut appartenir à quelqu'un et commence à traîner autour des parents de cette personne décédée ou ses descendants. Cela rend malade la personne qu'il choisit » (1993, p. 191). Les basses classes ou les esclaves étaient aussi comparés à des chiens errants (Suttles 1987, p. 6). Les traductions sont de l'auteur. 
activités rituelles envisagent une relation assez pacifiée avec l'être surnaturel, proche de celle entretenue avec un ange gardien, mais les anciens continuent de décrire des entités dangereuses et potentiellement hostiles avec lesquelles il est possible d'engager un combat pour la survie. Ces discours sont aussi mobilisés pour mettre à l'épreuve la détermination des initiés qui s'engagent dans les activités rituelles.

\section{L'entité surnaturelle comme propriété essentielle du soi}

Loin d'être véritablement extérieure à soi, l'entité surnaturelle peut aussi être perçue comme une partie de l'âme, et même porter les propriétés essentielles de cette âme. Dans ce cas, l'individu et l'entité participent du même flux vital. Esprits et humains peuvent donc partager ce flux, dont l'on peut tenter ici de mettre en lumière quelques propriétés.

La plupart du temps les entités surnaturelles sont désignées par le terme « esprit » (spirit). Par exemple, un soir, à la sortie d'une hutte de sudation, l'ancien qui avait dirigé la cérémonie confia à tous les participants que l'« esprit de l'ours » (bear spirit) était entré dans la loge de sudation durant un chant et qu'il avait eu une vision de l'ours. Il lui était venu des images d'ours et il avait senti sa présence. Qu'est-ce qui est donc désigné par ce terme d' « esprit de l'ours » ? Est-ce celui d'« un » ours particulier dont l'âme aurait pu errer dans les environs? L'esprit de l'ours qui est commun à tous les ours? L'ancien ne semblait pas faire de différence entre l'ours qui pouvait être rencontré en forêt et l'ours apparu dans la vision. Il semblait s'agir d'une sorte d'occurrence ou de token particulier. Ce token, qui peut apparaitre en rêve ou en forêt, est le signe envoyé par un esprit.

Lors d'une quête de vision, il faut être attentif et réceptif à tous les signes qui surgissent, notamment à la présence récurrente de tel animal particulier près de soi. Que ce soit en rêve, en vision ou de manière ostensive lors d'une marche en forêt, la présence d'un animal sera le plus souvent interprétée comme l'envoi d'un émissaire particulier, en lien avec l'esprit de l'espèce, c'est-à-dire comme une sorte de « représentant » intangible de l'espèce, souvent pensé et représenté sous la forme d'un prototype. Si un individu est en relation avec tel animal, il est en réalité en relation avec tous les individus qui sont de sa « sorte », c'est-à-dire qui possèdent ses mêmes propriétés essentielles. Il est donc possible d'interpréter la nature de l'entité surnaturelle comme une forme particulière de concept « sortal ». Wiggins définit ainsi ce qu'il entend par concept ou prédicat sortal : « N'importe quel prédicat dont l'extension consiste en tous les objets ou substances particuliers d'une espèce particulière, disons les chevaux ou les moutons ou les serpettes, sera appelé un prédicat "sortal". » (Wiggins 1980, p. 7). 
Comment penser le type d'extension du concept sortal utilisé pour se référer à l'entité protectrice ou esprit? Tout d'abord, comme nous venons de le voir, même si elle se présente sous la forme d'un animal, via un token, l'entité protectrice n'est jamais purement un animal. C'est un « esprit» (spirit). Par ce terme, il faut entendre qu'elle possède simultanément des propriétés mentales et physiques appartenant aux humains et aux non-humains. Deux témoignages permettent de mieux déterminer ce point.

Alors que nous discutions des quêtes de vision, une femme salish me fit part d'une vision pendant laquelle elle avait vu sa grand-mère décédée quelques années plus tôt : la vision l'avait fait pleurer lorsqu'elle l'avait reçue et avait suscité une grande émotion. Depuis, elle sentait que sa grand-mère la protégeait et qu'elle était liée à elle par des liens profonds. Dans sa vision, d'autres ancêtres s'étaient tenus à côté de sa grand-mère et elle avait senti sous la forme d'une certitude ineffable qu'elle était reliée à tous ces gens. À une autre occasion, cette femme m'avait déjà dit qu'elle était reliée à sa grand-mère et que cette dernière lui avait transmis le respect pour les oiseaux, dont les aigles. Sa grandmère était liée aux aigles. Il n'était pas anodin pour elle de participer à des cérémonies de sudation dans une loge placée sous le patronage de l'aigle et de la compassion. C'est donc à travers les émotions attachées à une aïeule qu'elle tissait un lien particulier aux aigles. Dans ce cas, l'esprit-ancêtre possédait des propriétés à la fois humaines et non humaines.

Une seconde tendance permettant de mieux concevoir la nature hybride des entités surnaturelles se manifeste dans les réponses d'un ancien très impliqué dans les activités rituelles. Quand je l'interrogeai sur ce qu'il pensait qu'il y avait après la mort, il me répondit que l'on se « réincarnait » dans d'autres êtres, comme un animal ou une plante avec lequel on avait un lien : « Les arbres sont des ancêtres. C'est pour cela qu'il faut autant les respecter. » Je lui demandai alors s'il croyait que nous étions tous reliés intimement à des animaux ou à des plantes. Il ne le savait pas avec certitude, mais il le croyait : si un individu pouvait se réincarner en un autre être, c'est qu'il devait bien y avoir un lien entre eux.

Sans être explicité, ce point de vue rejoint fortement les conceptions classiques des Salish centraux de la côte, selon lesquelles tous les êtres descendent d'ancêtres hybrides mi-humains, mi-non humains. À l'origine, la région était peuplée d'êtres humanoïdes, sujets à des transformations perpétuelles, généralement en animaux : il leur suffisait d'ôter leur peau animale et de redevenir humain. Chacun de ces êtres humanoïdes possédait des propriétés comportementales et un nom proches de ceux d'une espèce particulière. Leur forme instable fut réparée (fixed) par un ou des transformateurs (khaals ou xals) (voir, entre autres, Suttles et Jenness 1956 ; Carlson et McHalsie 2001, 1998, p. 29-30 ; Carlson 1997). Les individus contemporains descendent en réalité d'un ancêtre 
totémique commun qui possède des propriétés mixtes, humaines et animales, et qui est parfois lié à un lieu géographique précis. Il ne s'agit pas seulement de relations entre humains et animaux ou végétaux, mais aussi avec des éléments du paysage. Ce qui caractérise le mieux la nature de ces êtres surnaturels est qu'ils ne sont ni complètement humains, ni complètement animaux, et qu'ils possèdent une force de vie qui est à l'origine d'humains (individus ou groupes) et de non-humains (individus ou groupes).

Les humains sont donc reliés à d'autres espèces de non-humains par un être mythique qui serait leur commune origine. Cette configuration totémique est par exemple précocement repérée chez les Salish centraux de la côte par HillTout à la fin du XIX ${ }^{\mathrm{e}}$ siècle : un de ses informateurs, François, lui expliqua que « même s'ils croyaient que leur ancêtre éloigné fut une loutre, il ne pensait pas que c'était le même genre de loutre qui vit de nos jours. Les loutres desquelles ils descendent étaient des gens-loutres, pas des animaux, qui avaient le pouvoir de changer leur forme d'hommes et de femmes en celle de la loutre. Tous les animaux lors des temps anciens étaient ainsi. Ce n'était pas juste des animaux communs et rien de plus [...]», et Hill-Tout de continuer : " ces ancêtres ne sont pas regardés comme de simples animaux mais comme ayant ou prenant part aussi à des natures duelles » $(1978$, p. 110 ; traduction et italiques de l'auteur). De nos jours, ces ancêtres totémiques peuvent être rappelés par des familles, sous l'égide de relations filiales lointaines. J'ai pour ma part entendu des individus rappeler des liens familiaux avec le saumon, l'oiseau-tonnerre et l'ours. Une cartographie plus précise des liens familiaux totémiques est proposée par McHalsie, regroupant le saumon sockeye, le grizzly, le pic-vert à tête rouge, le cèdre, le vison, l'herbe coupée, le colibri, la grenouille géante, la salamandre, le geai bleu, etc. (Carlson et McHalsie 2001, p. 25). L'entité surnaturelle animale ou végétale, l'ancêtre totémique protecteur, possède donc des ensembles de propriétés ontologiques morales et physiques hybrides qui permettent d'assurer le lien entre l'humain et son animal protecteur.

L'esprit (spirit) peut donc être dit lié à une partie essentielle de l'individu, en tant qu'il partagerait avec lui une sorte de flux vital idiosyncratique. Mais comment penser ce flux, ces paquets partagés de propriétés ontologiques essentielles? Il est possible de considérer que la manière d'identifier ces propriétés se fait en les comprenant comme trouvant leur origine dans un ancêtre surnaturel, commun à la fois à l'humain et au non-humain. Cet ancêtre est ainsi au cœur de l'identification totémique. Cette identification, sur laquelle repose l'idée que l'humain partage des propriétés essentielles avec l'entité surnaturelle, n'est pas l'affirmation d'une identité directe ( « individu $\mathrm{x}=$ animal y », par exemple la proposition « Je suis un ours »). La règle d'identification totémique, lorsque l'entité surnaturelle est au cour du soi, semble se construire par la médiation d'un concept « sortal » indéterminé (dont le paradigme est l'ancêtre commun). 
Dès lors, l'identité partagée par un individu humain et un individu non humain d'une autre espèce (par exemple, un ours) peut être décrite ainsi : $x=F, y=F$ (où $\mathrm{x}$ et $\mathrm{y}$ sont l'humain et le non-humain et $\mathrm{F}$ l'ancêtre commun), et non pas par une relation d'identité directe du type $\mathrm{x}=\mathrm{y}$ (où $\mathrm{x}$ et $\mathrm{y}$ sont l'humain et le non-humain). Cette identification sortale (F) permet ainsi de penser le paquet de propriétés ontologiques partagé à la fois par l'humain et le non-humain, mais aussi les transformations propres aux individus (ce qui reste du soi après la mort, par exemple). Ainsi, l'individu qui devient un ours après la mort ne fait peut-être que réaliser deux états de sa propre substance. Il apparaît ainsi, comme le suggère Guédon dans sa réflexion sur les entités surnaturelles de la côte nord-ouest, que « la transformation n'est pas tant un processus qu'une qualité correspondant à de multiples identités ou de multiples points de vue ou réalités que l'on attribue à une entité particulière » (Guédon 1984, p. 142).

\section{Vers une force de vie surnaturelle diffuse}

L'hésitation constitutive des relations que l'on entretient avec une entité surnaturelle permet de circonscrire certains schèmes récurrents d'identification : il s'agit d'une partie de soi qui est souvent conçue sous la forme d'une relation profonde de filiation ou d'héritage de propriétés déjà possédées par ses aïeuls, mais aussi d'un lien d'apprivoisement, d'alliance, fondé sur la rencontre de deux pouvoirs distincts, humain et non humain. Ces relations nous conduisent vers une appréhension des propriétés ontologiques de l'entité surnaturelle comme mixte hybride de propriétés humaines et non humaines, ce qui coïncide encore de nos jours avec les représentations des entités surnaturelles présentes dans les rapports ethnographiques du début du Xxe siècle. Il s'agit d'existants humanoïdes pouvant se transformer en animaux. En réalité, qu'il s'agisse d'une force extérieure à soi ou d'une force interne, constitutive du soi, de nombreuses justifications concernant l'entité protectrice mobilisent l'idée d'une « force spirituelle» (spiritual power) qui se donne sous la forme d'une contagion diffuse.

Une jeune mère, originaire de l'île de Vancouver, avait invité quelques amis à une petite cérémonie dans le jardin d'une maison de la banlieue sud, pour nous présenter la danse de l'esprit du colibri (hummingbird) qu'elle avait reçue en rêve quelques semaines auparavant. L'esprit du colibri lui avait recommandé de faire une danse, en lui montrant les pas et le chant qui étaient propices à sa manifestation. L'esprit avait ajouté qu'il les protégerait, elle et sa petite fille de cinq ans, et que pour cela elle pouvait lui rendre hommage. La jeune mère exécuta la danse et le chant avec sa petite fille. Leur danse était accomplie les bras perpendiculaires au corps, par petits bonds, ce qui pouvait rappeler la posture et les mouvements du colibri, sans pour autant que l'entité soit totalement reconnaissable. L'implication de l'enfant montre que l'entité protectrice est 
certes reçue de manière personnelle et intime par la mère, mais qu'elle peut aussi tenir lieu de lien totémique intergénérationnel entre la mère et sa fille. De même, cet esprit apparaît comme extérieur - il a besoin d'une danse pour être remercié -, mais en même temps il contribue à la représentation du lien singulier et unique qui attache la mère et la fille. La même « force spirituelle », celle de l'esprit du colibri, traverse donc à la fois la mère, la fille et les colibris. Enfin, le fait de rendre cette danse publique suggère que la seule relation intime, bien que suffisante, doit pouvoir entrer dans une économie sociale et généralisée des relations avec les non-humains surnaturels.

L'esprit du colibri est d'abord une force spirituelle (spiritual force) - expression souvent utilisée pour parler des esprits. Cette danse privée suit un modèle ancien de présentation publique du lien créé avec une entité surnaturelle : des cérémonies, de grande ampleur ou en petit comité, nommées « danses d'hiver » ou « danses spirituelles », sont organisées, principalement en hiver, afin que les individus puissent témoigner du lien qu'ils ont noué avec une entité surnaturelle (sur les danses spirituelles voir notamment Wike 1941 ; Robinson 1963 ; Kew 1970 ; Jilek 1974 ; Amoss 1978 ; Bierwert 1999).

Si autrefois les individus en transe lors des danses d'hiver entraient en contact avec des entités protectrices distinctes (Wike 1941), de nos jours il est plutôt dit qu'ils se laissent posséder par une force spirituelle diffuse, que les Salish du haut et moyen Fraser nomment syowen (Kew 1970 ; Jilek 1974 ; Bierwert 1999). Cette force spirituelle est décrite en termes d'un pouvoir qui n'est pas rapporté à une entité animale ou végétale particulière, comme cela était le cas autrefois, mais qui possède les caractéristiques de la protection et du danger spirituel propres aux entités totémiques. Frank Malloway, leader stó:lō, s'adressant à l'anthropologue Biewert dans les années 1990, dit ainsi à propos du syowen : « Et il en est ainsi - le syowen est une force étrange, et, lorsque tu commenceras à penser que tu la connais, tu sais, cela te fera prendre conscience qu'en réalité tu ne sais rien. Cela fera quelque chose... Je l'ai fréquenté (presque toute ma vie) et je ne connais presque rien sur sa nature. Je ne peux pas le décrire. Je ne peux pas dire à quelqu'un ce que c'est, parce que mes mots feront demi-tour et deviendront quelque chose d'autre. Mais il te stupéfait toujours chaque hiver. Quelque chose se passe. » (Bierwert 1999, p. 196 ; traduction de l'auteur). Aujourd'hui cette force est moins personnelle (elle est la même pour tous les individus) et moins liée à un animal, mais elle possède la même nature protectrice et dangereuse que les entités totémiques. Même si cette force est plus impersonnelle qu'un non-humain surnaturel, elle est tout de même appréhendée à travers de nombreux traits qui lui confèrent un caractère relativement individualisé : elle est jalouse, taquine et possessive (ibid., p. 180).

Il est aussi important de relever que cette idée d'une possession par une « force spirituelle diffuse et impersonnelle » fut au cœur de la religion des Salish côtiers 
nommée « shakerisme » (voir entre autres, Barnett 1957; Amoss 1990 ; Ruby et Brown 1996 ; Neylan 2011). Cette religion syncrétique, entre christianisme et croyances locales, naquit sur le territoire des Salish de la côte (Sahewamish [Squaxin]) en 1881. Le prophète, John Slocum (Squ-Sacht-um), reçut la révélation d'un remède offert par Dieu : il parvint à faire passer le « pouvoir » de Dieu en tremblant de manière incontrôlable et à guérir. Ainsi, l'église shaker organisait de grandes messes où les individus pouvaient entrer en contact avec cette « force-qui-fait-trembler », via le pouvoir de l'Esprit saint. La force diffuse et impersonnelle du Saint-Esprit a donc été très tôt interprétée comme la présence d'un être surnaturel, sur le modèle de ceux rencontrés dans les quêtes de vision : sa principale caractéristique était de faire trembler, tout comme ce fut toujours le cas, chez les Salish côtiers, dans les comptes rendus de rencontres avec les entités surnaturelles (Wells 1987 ; Mohs 1994, p. 195). Le shakerisme entretient donc des liens étroits avec les danses spirituelles d'hiver, durant lesquelles les individus possédés par le pouvoir diffus du syowen entrent en transe et tremblent : dans ce cadre, le rite ne retient du rapport à l'entité totémique que l'idée d'un « pouvoir ${ }^{10}$. L'étude de la lente évolution des danses d'hiver laisse paraître la transformation du rapport des initiés à leur entité totémique singulière (Wike 1941 ; Robinson 1963) en une force de plus en plus diffuse et impersonnelle, le syowen (Kew 1970 ; Jilek 1974 ; Amoss 1978 ; Bierwert 1999).

De nombreux autochtones sont aujourd'hui catholiques ou protestants pratiquants et certains préfèrent parler d'une relation particulière au Saint-Esprit. Cette force garde des aspects personnels : elle apparaît souvent comme la manifestation du pouvoir vital de Dieu ou du Grand Esprit. Ainsi, l'autel de la petite église Saint-Paul, sur la réserve Squamish, au nord de Vancouver, est entouré de branches sacrées de cèdre et les pichets qui contiennent le vin eucharistique sont ornés d'aigles autochtones. La force impersonnelle eucharistique oscille donc souvent entre celles de l'Esprit-Saint, du Grand Esprit ou d'entités totémiques singularisées, proches de la colombe, comme l'aigle.

\section{De l'identification intime avec un non-humain au complexe totémique}

Des exemples que nous avons présentés jusqu'ici se dégagent déjà cinq rapports différents et singuliers aux non-humains surnaturels.

1. Un totémisme individuel classique : la prérogative de l'identification totémique chez les Salish centraux de la côte est d'abord donnée à la rencontre personnelle et privée d'un « individu humain avec le représentant d'une espèce non humaine », que ce soit lors d'une rencontre tangible, d'un rêve ou d'une

10. Un rapprochement entre l'esprit diffus qui fait trembler le danseur dans le shakerisme et les cérémonies de danses d'hiver est aussi proposé par Neylan (2011, p. 195-196). 
quête de vision. Par exemple, tel individu entre en contact avec l'esprit de l'ours, de la loutre, de l'aigle, etc.

2. Un totémisme collectif classique : nous avons vu qu'une place importante est accordée à la relation d'un groupe humain avec une espèce animale ou végétale. Tous les individus d'un groupe humain déterminé auront dès lors une relation particulière avec tous les individus d'un autre groupe non humain. Par exemple, telle famille est liée au saumon ou tel groupe de danseurs est lié à l'aigle, à l'ours, etc. Ainsi en est-il du colibri qui lie la jeune mère et sa petite fille.

3. Un totémisme individuel singulier : il est des cas où, lors de quêtes de visions, un individu ne rencontre pas un membre d'une espèce, mais une entité non humaine parfaitement singulière. C'est le cas, par exemple, de certaines créatures comme l'oiseau-tonnerre.

4. Un totémisme collectif singulier : dans certains cas encore, un groupe humain - une famille ou un ensemble d'individus pratiquant un rituel - peut aussi entretenir une relation totémique avec une entité individuelle et singulière qui n'est pas la représentante d'une espèce. C'est le cas, par exemple, de certaines familles musqueam avec l'oiseau-tonnerre mais aussi avec le serpent-à-deux-têtes.

5. Un totémisme indistinct et flottant : de nombreux individus rapportent l'expérience d'un contact avec une force impersonnelle et surnaturelle. Le parangon de cette relation particulière parmi les Salish centraux de la côte est le syowen, une force spirituelle et vitale qui traverse tous les individus qui entrent en contact avec le monde spirituel et qui peut avoir des effets concrets sur les corps, tels le tremblement et la transe.

Derrière les hésitations et les incertitudes, certaines explications reviennent donc de manière répétée dans les discours. Il est possible de nommer « complexe totémique » la cohabitation dans la région de ces relations plurielles aux nonhumains surnaturels. Malgré leur hétérogénéité elles peuvent être organisées selon une logique qui permet de les replacer à l'intérieur d'un même « complexe », c'est-à-dire une structure qui met en lumière leur rapport.

\section{Vers un complexe totémique propre aux Salish centraux de la côte}

Le totémisme est pensé depuis Lévi-Strauss (1962a et b) comme un système de codage permettant d'organiser le monde à partir de saillances entre propriétés universelles et propriétés particulières. Cette organisation est selon lui possible grâce à un outil mental découpant et ordonnant les différences perceptuelles, outil qu'il nomme « opérateur totémique » (1962b, p. 184). Cet opérateur permet de découper le réel en parties manipulables et d'organiser à partir de ces mêmes saillances des regroupements d'êtres. Dans Le totémisme aujourd'hui, Lévi-Strauss propose quatre formes possibles de cette liaison entre l'universel et le particulier, qui sont pour lui repérables à différents endroits du monde (1962a, p. 27). 


\begin{tabular}{|c|c|c|c|c|}
\hline Nature & Catégorie & Catégorie & Individu & Individu \\
\hline Culture & Groupe & Personne & Personne & Groupe \\
\hline
\end{tabular}

Fig. 1 - Les différentes opérations totémiques selon Lévi-Strauss.

Il s'agit toujours, que ce soit au niveau de la nature ou à celui de la culture, de penser un lien logique entre la partie (personne/individu) et le tout (groupe/ catégorie). Simplifions donc le tableau proposé par Lévi-Strauss pour arriver au cœur du processus de particularisation et d'universalisation au fondement $\mathrm{du}$ totémisme. Les termes de « groupe » et de « catégorie » renvoient en réalité à la même opération cognitive d' " universalisation » et ceux d' " individu » et de " personne » procèdent de la même opération logique de « particularisation ». Substituons encore aux deux concepts « individu » et « personne », celui unique d' " individu », et à ceux de « groupe » et de " catégorie », qui dénotent des extensions d'individus particuliers, celui unique de «groupe ». D'un côté, un individu humain peut entrer en contact avec un groupe de non-humains (l'ensemble des loutres), d'un autre côté, un groupe humain peut entrer en contact avec un groupe de non-humains (l'ensemble d'une famille humaine vs l'ensemble des loutres). La seconde opération de simplification consiste à remplacer la dichotomie nature/culture, par la distinction moins spécifique entre « non-humain » (monde naturel) et « humain » (monde culturel). La simplification proposée permet maintenant de ranger un certain nombre de combinaisons de rapports entre les humains et les non-humains, que le mot de « totémisme » pourrait contenir (voir Figure 2, page suivante).

Loin de représenter des formes éparses à travers le monde, ces quatre opérations de rapport individu/groupe devraient pouvoir représenter la cristallisation des hésitations propres à définir la relation d'un humain et d'un non-humain surnaturel, et cela à l'intérieur de justifications fragmentaires et confuses. Il serait ainsi possible de considérer que le « complexe totémique » émerge d'une oscillation, d'incertitudes ou d'hésitations signifiantes quant à la relation qu'un humain ou qu'un groupe d'humains doit entretenir avec un individu ou un groupe non humain surnaturel. Ces quatre opérations logiques se repèrent clairement dans les justifications et les pratiques des Salish centraux de la côte.

Si nous considérons donc que le totémisme est bien un outil de classification, reposant sur une discrimination minimale entre l'universel et le particulier, le collectif et l'individuel, il se déploie en cinq formes logiques chez les Salish centraux de la côte. Ce carré doit pouvoir se lire comme une carte d'analyse de différentes justifications de pratiques sociales, prenant la forme de groupes de transformation (voir Figure 3, page suivante). 


\begin{tabular}{|c|c|c|c|}
\hline & & \multicolumn{2}{|c|}{ Non-humains } \\
\hline & & Individu & Groupe \\
\hline \multirow{4}{*}{.气气 } & $\vec{z}$ & $\begin{array}{l}\text { Totémisme individuel } \\
\text { singulier }\end{array}$ & $\begin{array}{l}\text { Totémisme individuel } \\
\text { classique }\end{array}$ \\
\hline & 雍 & $\begin{array}{l}\text { Rencontre d'un individu humain } \\
\text { avec un individu particulier } \\
\text { d'une espèce non humaine. }\end{array}$ & $\begin{array}{l}\text { Rencontre d'un individu } \\
\text { avec l'esprit d'une espèce ou } \\
\text { le « maître » d'une espèce. }\end{array}$ \\
\hline & & Totémisme collectif singulier & Totémisme collectif classique \\
\hline & 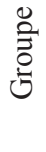 & $\begin{array}{l}\text { Rencontre d'un groupe } \\
\text { humain avec un individu } \\
\text { non humain singulier. }\end{array}$ & $\begin{array}{c}\text { Rencontre d'un groupe } \\
\text { d'humains avec l'esprit } \\
\text { d'une espèce ou un « maître » } \\
\text { d'une espèce. }\end{array}$ \\
\hline
\end{tabular}

Fig. 2 - Le complexe totémique.

\begin{tabular}{|c|c|c|c|}
\hline & & \multicolumn{2}{|c|}{ Non-humains } \\
\hline & & Individu & Groupe \\
\hline \multirow{4}{*}{ } & : & $\begin{array}{c}\text { Stl'aleqem } \\
\text { Totémisme individuel singulier } \\
\text { Un individu rencontre une } \\
\text { créature unique comme } \\
\text { l'oiseau-tonnerre, le sasquatch, } \\
\text { le serpent-à-deux-têtes ou } \\
\text { les bébés-des-eaux. }\end{array}$ & $\begin{array}{c}\text { Sulia (syuwél) } \\
\text { Totémisme individuel classique } \\
\text { Un individu rencontre un } \\
\text { esprit, représentant d'une } \\
\text { espèce, comme l'ours, } \\
\text { le cerf, la loutre, etc. }\end{array}$ \\
\hline & & \multicolumn{2}{|c|}{ Sociétés secrètes SYOWEN } \\
\hline & & Totémisme collectif singulier & Sululia (syweals) \\
\hline & 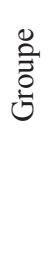 & $\begin{array}{l}\text { une créature unique (une entité } \\
\text { protectrice particulière comme } \\
\text { l'oiseau-tonnerre ou le serpent- } \\
\text { à-deux-têtes que l'on retrouve } \\
\text { dessiné sur le pôle d'une maison } \\
\text { de cérémonie à Chilliwack). }\end{array}$ & $\begin{array}{l}\text { Une famille est liée par } \\
\text { filiation à un ancêtre des } \\
\text { origines, porteur des propriétés } \\
\text { d'une espèce comme l'ours, } \\
\text { le saumon, l'aigle, etc. }\end{array}$ \\
\hline
\end{tabular}

Fig. 3 - Le complexe totémique des Salish centraux de la côte.

Ces différentes formes ont été définies par l'examen des identifications totémiques contemporaines. Ces justifications du lien aux entités totémiques apparaissent ainsi comme des reformulations de pratiques plus anciennes chez les Salish côtiers, qui nous sont rappelées par la persistance d'un certain nombre de termes halkomelem vernaculaires. 
1. Un totémisme individuel classique (entités sulia ou syuwél) : un individu humain entre en contact avec le représentant d'une espèce non humaine. Ces créatures surnaturelles protectrices sont aujourd'hui désignées par le mot halkomelem syuwél, qui désigne à la fois l'esprit protecteur et le chant offert par cet esprit protecteur (Suttles et Jenness 1956, p. 6 ; Galloway 1994, p. 491). Même si peu d'individus parlent aujourd'hui couramment l'halkomelem, ce terme est aujourd'hui encore utilisé à l'intérieur d'énoncés en anglais pour désigner l'esprit protecteur.

2. Un totémisme collectif classique (entités sululia ou syewà:ls) : cette forme désigne la relation d'un groupe humain avec une espèce animale ou végétale. Ces entités surnaturelles sont souvent prises dans une relation de parenté, comme des ancêtres des origines. Même si de nos jours la différence nominale n'est plus faite entre les esprits protecteurs personnels et les esprits reçus en héritage, il est possible de remarquer dans la littérature ethnographique que les anciens Stó:lō s'y réfèrent sous le terme syewà: $l s$, quelque peu différent de syuwél (Wells 1987, p. 94-95). Le lien est aussi établi par Hill-Tout (1978, p. 110) à maintes reprises dans sa distinction entre sululia (ancêtre mythique familial) et sulia (esprit protecteur individuel).

3. Un totémisme individuel singulier (entités stl'áleqem) : dans ce cas particulier de totémisme, un individu ne rencontre pas un membre d'une espèce, mais une entité non humaine parfaitement singulière. Ces créatures particulières, comme l'oiseau-tonnerre, sont souvent rangées par les anciens dans la catégorie des êtres stl'áleqem. Il n'y a dans cette catégorie que des entités surnaturelles uniques et singulières qui ne sont pas les délégués ou les représentants d'une espèce. Par exemple, le serpent-à-deux-têtes, les yeux-flamboyants, l'asticot, l'ours-noir-vivant-sous-les-eaux, etc. (pour une description de ces entités, Carlson et McHalsie 2001, p. 8 ; Hill-Tout 1978, p. 73-76 ; Van Eijik 2001, p. 179 ; Suttles 1987, p. 75-76 ; Wells 1987, p. 54-55, 84-85 et 156-157).

4. Un totémisme collectif singulier (entités stl'áleqem) : un cas de totémisme collectif est celui où un groupe humain - une famille ou un ensemble d'individus pratiquant un rituel (société secrète) - peut aussi entretenir une relation totémique avec une entité individuelle et singulière qui n'est pas la représentante d'une espèce. Ce cas de totémisme, un peu plus rare chez les Salish centraux de la côte, est celui au travers duquel des familles entières se disent liées à l'oiseautonnerre, qui est une créature unique. Dans la littérature ethnographique, les cas de protection de groupes de personnes par une entité unique et singulière sont davantage présents dans les descriptions des sociétés secrètes des voisins septentrionaux des Salish côtiers, les Kwakwaka'wakw (Kwakiutl) : la plus prestigieuse de ces sociétés secrètes est Hamatsa, dont les membres entretiennent une relation avec Baxbaxwalanuksiwe, un géant mangeur d'hommes.

5. Un totémisme indistinct et flottant (syowen) : beaucoup d'individus rapportent un contact avec une force impersonnelle et surnaturelle dont le parangon est le syowen. Ce terme est présent dès les premières études ethnographiques 
sur les danses spirituelles d'hiver des Salish centraux de la côte (Wike 1941 ; Robinson 1963 ; Kew 1970 ; Jilek 1974 ; Amoss 1978 ; Bierwert 1999) et prend aujourd'hui plus d'importance que les identifications totémiques singulières, qui étaient autrefois plus prégnantes lors des danses d'hiver (Wike 1941).

Parmi cet ensemble de relations totémiques, la notion de syowen, force de vie diffuse, semble opérer sur le modèle d'un signifiant flottant, d'un retour à la seule présence du surnaturel comme force spirituelle (spiritual power), au-delà des formes physiques qu'il pourrait revêtir. Chacun voit ainsi dans le syowen ce qu'il y met. Le syowen peut ainsi être pensé comme un opérateur de passage, comme cette case vide à laquelle Lévi-Strauss fait référence pour désigner les termes religieux englobants de mana ou de wakan (Lévi-Strauss 1950). C'est ainsi, comme tout signifiant flottant, un terme permettant de définir le monde surnaturel comme réunion des contraires : il s'agit d'une force qui est à la fois personnelle et impersonnelle, qui est protectrice et dangereuse, individuelle et collective, et qui permet de faire des liens avec le concept d'Esprit saint, la notion pan-autochtone de Grand Esprit ou encore des notions plus contemporaines et new age comme l'énergie. Cette notion de force diffuse, qui est aujourd'hui au cœur des justifications des danses d'hiver, permet donc de réunir symboliquement la plupart des caractéristiques et formes de totémisme présentes chez les Salish centraux de la côte : son caractère extrêmement vague permet d'éviter tout discours de spécification de la nature de l'entité avec laquelle on est en contact et du mode de relation que l'on entretient avec elle. Le syowen cristallise cette opération totémique en tant qu'elle est toujours fluctuante et hésitante dans la désignation d'un rapport particulier à un non-humain surnaturel souvent incompréhensible et indescriptible.

Essai de résolution des problèmes ethnographiques concernant la nature du totémisme des Salish centraux de la côte

Ces considérations sur le totémisme contemporain des Salish centraux de la côte permettent peut-être de reformuler les termes du débat centenaire concernant la proximité entre totémisme individuel et totémisme collectif dans la région. Les premières données ethnographiques systématiques, recueillies vers la fin du XIX ${ }^{\mathrm{e}}$ siècle et au début du XX $\mathrm{X}^{\mathrm{e}}$ siècle, principalement par l'école boasienne, s'appuient sur un concept de totémisme défini a minima par Frazer comme « une identification entre un homme et son totem, que ce soit un animal ou une plante, ou quelque chose d'autre » (Frazer 1887, p. 3 ; traduction de l'auteur). Mais dès 1897, dans son article intitulé The social organization and the secret societies ot the Kwakiutl Indians, Boas se méfie des grandes catégorisations sociologiques. Il réitérera cette méfiance dans un article de 1916, « The origin of totemism » : « Je ne pense pas que tous les phénomènes totémiques puissent être des formes dérivées des mêmes sources psychologiques et historiques. 
Le totémisme est une unité artificielle et non naturelle. » (Boas 1916, p. 321 ; traduction de l'auteur). Le totémisme Kwakiutl ou Salish a selon lui peu à voir avec le totémisme australien des Arunda, notamment parce que le totémisme clanique salish prend racine dans une forme primitive de totémisme individuel (voir aussi sur ces questions, les considérations de Goldenweiser 1910).

En 1904, Hill-Tout publie « Totemism: a consideration of its origin and import ». Il y considère que dans « dans ce pays [la Colombie-Britannique], la majorité des chercheurs professent l'opinion selon laquelle le totem "clanique" n'est qu'un développement social du totem individuel [...]. Ils sont irrésistiblement conduits à cette conclusion par les données à leur disposition » (Hill-Tout 1904, p. 71 ; traduction de l'auteur). Le totémisme clanique serait ainsi régulièrement conçu comme une dégénérescence du totémisme individuel. L'anthropologie de la côte Nord-Ouest est donc prise dans un double mouvement : elle repère une certaine relation d'antériorité du totémisme individuel par rapport au totémisme clanique, soulignant la relation particulière entretenue entre un humain et un esprit, et, en même temps, elle opère une forme de désuniversalisation du phénomène totémique.

Pour Hill-Tout (1978, p. 85), qui a longuement travaillé avec les Salish centraux de la côte, le totémisme est loin d'être un simple instrument de classification de groupes sociaux. Le « totem » est avant tout une « vision » intime : il y a une précédence du totémisme individuel sur le totémisme collectif. Pour le démontrer, il utilise deux arguments. C'est la racine du mot « rêver » qui forme le terme " entité surnaturelle » chez les Salish côtiers. Il s'agit donc toujours d'abord d'une rencontre privée car on ne peut rêver à plusieurs. Ce n'est que dans un second temps que ces entités sont transmises. De plus, le totémisme ne repose pas sur une structure clanique car il a survécu au passage de formes d'organisation matrilinéaires à des formes patrilinéaires. Cela montre bien qu'il survit à la destruction des structures sociales et qu'il désigne plutôt une relation privée et intime. Développons brièvement ces deux points.

Hill-Tout s'appuie sur l'étymologie du mot sulia (esprit protecteur, aujourd'hui écrit syuwél) dans les différents dialectes halkomelem et remarque que la racine /ulia/ signifie " rêver ». Ces êtres apparaissent avant tout comme des êtres rencontrés dans des rêves ou visions - états mentaux qui sont toujours personnels et privés. Le monde personnel du rêve ou de la vision est donc toujours le premier lieu de contact. Il est courant de constater que la vision d'un seul individu (totémisme individuel) peut affecter des générations entières (totémisme collectif). Un informateur de Hill-Tout, Dr. George, lui raconta que lorsque son grand-père rencontra son esprit protecteur (un ours) au cours d'une chasse, ce dernier lui annonça qu'il protégerait aussi les membres de sa famille et de sa descendance. Toute la famille était désormais connectée par cet incident (ibid., p. 111). Selon les informateurs chehalis de Hill-Tout, seuls les chamanes pouvaient acquérir leur sulia de leurs pères, alors que tous 
les autres hommes devaient aller les acquérir par eux-mêmes, lors de quêtes de vision. Il est dès lors fort concevable - comme le propose Hill-Tout - de considérer que toutes les relations de protection ont pour origine une vision individuelle et que le totémisme collectif est une forme altérée du totémisme individuel. Hill-Tout fait remarquer qu'il n'est pas certain de la possibilité de la transmission d'un esprit protecteur et que sur cette question les autochtones sont largement « divisés et en contradiction » (ibid.). Ainsi, pour les premiers ethnologues ayant étudié le totémisme des Salish centraux de la côte, un «choix culturel $\gg{ }^{11}$ semble toujours à l'origine de la présence marquée d'un de ces deux totémismes, individuel ou collectif. Hill-Tout montre par exemple que chez les Thompson les totems héraldiques sont en général très peu connus, alors que chez les Halkomelem du Fraser les armoiries apparaissent sur les poteaux du fronton des maisons. De ce fait, pour Hill-Tout, le totémisme collectif et le totémisme individuel « existaient parmi les Salish » (ibid., p. 113).

Le totémisme ne se déploie pas pour autant en une infinité de formes possibles car il organise les combinaisons individuelles et collectives à partir d'un double découpage : celui de l'individu et du groupe, celui des humains et des non-humains. L'analyse proposée ici du « complexe totémique » est donc non seulement en concordance avec les réflexions de Hill-Tout sur le totémisme propre aux Salish côtiers, mais elle se situe aussi dans le prolongement des propositions de Lévi-Strauss (1962a et b) qui voit dans le totémisme un ensemble encodé de relations entre les humains (culture) et les non-humains (nature) à partir de saillances organisées selon les rapports de l'individu et du groupe.

Dans ses récentes propositions de reprise du concept de totémisme, Descola (2005) resserre les caractéristiques propres au totémisme afin d'en faire une unité ontologique, c'est-à-dire un système de distribution de paquets de propriétés à l'ensemble des existants. La « formule ontologique du totémisme » (ibid., p. 232) opère donc par continuité interspécifique (entre humains et non-humains) de propriétés physiques et mentales, mais aussi à travers une identification cognitive et émotionnelle (c'est-à-dire un rapport intime) de paquets de saillances. Elle ne se résume donc pas à une simple méthode de classification et d'organisation, comme c'est le cas chez Lévi-Strauss. Une particularité de la définition descolienne du totémisme, qui la rend particulièrement adéquate à une application possible aux modes relationnels chez les Salish centraux de la côte, est que le rapprochement interspécifique entre humains et non-humains se construit par référence à des « êtres du Rêve » (sur le modèle australien), c'est-à-dire à des non-humains surnaturels qui possèdent initialement les mixtes substantiels partagés par les différents existants - ce que le présent article nomme « concept sortal » de l'entité hybride et qui constitue le cœur de l'identification totémique.

11. Hill-Tout parle de « sélection » et d' « adoption » (ibid., p. 82). 
En effet, chez les Salish centraux de la côte, comme nous l'avons vu, le rapport avec l'entité surnaturel se fait via un concept indéterminé de flux vital. Ce concept indéterminé est « sortal », au sens où il désigne d'abord une sorte ou une espèce (une force de vie ou un ancêtre commun). En ce sens, il permet de désigner le lien commun entre l'humain et le non-humain et les paquets de propriétés qu'ils partagent. Ce concept sortal permet ainsi, dans les justifications, de penser l'entité surnaturelle à la fois à travers un rapport extérieur au soi (une affinité singulière du fait de la détention de propriétés vitales partagées) ou à travers un rapport intérieur au soi (un flux vital trouvant son origine dans la parenté de l'humain et du non-humain avec un ancêtre commun surnaturel). Nous avons ainsi pu montrer que le complexe totémique, en tant qu'opérateur de liaison entre l'humain et le non-humain, ne se donne pas comme un rapport figé, mais comme une pluralité de combinaisons possibles, présente dans les tâtonnements, incertitudes et hésitations. On peut donc émettre l'hypothèse, comme le pensait Lévi-Strauss à l'échelle du monde entier, que le totémisme est toujours une pluralité irréductible des relations, mais qu'elles se présentent ici d'abord sous la forme de combinaisons à une échelle locale.

Les incertitudes et tâtonnements explicatifs sont donc essentiels chez les Salish côtiers pour exprimer sa relation à une entité surnaturelle. Pourtant l'étude de ces hésitations laisse paraître un nombre restreint de justifications relationnelles. Si l'on veut comprendre la possibilité du totémisme aujourd'hui, il faut tenir ensemble à la fois les hésitations (contenu négatif - ce que l'on ne sait pas) et les justifications (contenu positif - ce que l'on sait) : la notion de « complexe totémique », comme structure de transformation, permet ainsi de saisir l'ensemble de ces types de données ethnographiques. Cette réflexion entend apporter au débat deux idées essentielles. D'une part, le totémisme requiert, comme schème de rapport au monde, un concept sortal permettant de penser une identité hybride liant les existants et autorisant un certain nombre de rapports possibles à partir de la distribution de paquets de propriétés ontologiques. D'autre part, s'il est envisageable de concevoir la présence d'une « ontologie totémique » sur la côte Nord-Ouest, comme le propose Descola (2005), cette dernière ne peut être regardée comme une structure « figée » mais doit au contraire apparaître comme une construction pragmatique. Le complexe totémique n'est donc pas seulement un groupe de transformation abstrait, une structure faite pour des yeux d'anthropologue, il est d'abord ce lieu concret d'interactions où chacun justifie ses pratiques et ses ressentis, met à l'épreuve des modes de relations et construit des formes d'existence acceptables pour accueillir les entités qui peuplent son monde. *

* Manuscrit reçu en octobre 2013, accepté pour publication en octobre 2014.

Je souhaite remercier très chaleureusement Paul Sorrentino pour la relecture attentive et éclairante de cet article, ainsi que Daphné Le Roux et Camille Chamois, pour leurs précieux conseils. 


\section{Références citées}

Amoss Pamela

1978, Coast Salish spirit dancing: the survival of an ancestral religion, University of Washington Press, Washington.

1990, « The Indian Shaker church », in Northwest Coast. Handbook of North American Indians, Smithsonian Institution Press, Washington.

BARNETT Homer G.

1955, The Coast Salish of British Columbia, University of Oregon, Eugene.

1957, Indian Shakers: a messianic cult of the Pacific Northwest, Southern Illinois University Press, Carbondale.

BIERWERT Crisca

1999, Brushed by cedar, living by the river: Coast Salish figures of power, University of Arizona Press, Tucson.

BLOCH Maurice

2005, Essays on cultural transmission, Berg Publisher, Oxford.

BoAs Franz

1916, « The origin of totemism », American anthropologist, 18 (3), p. 319-326.

CARLSON Keith Thor (éd.)

1997, You are asked to witness: the Stó:lō in Canada's Pacific Coast history, Stól:lō Heritage Trust, Chilliwack.

Carlson Keith Thor, Albert Jules McHalsie et Kate Blomfield

2001, A Stó:lō-Coast Salish historical atlas, Douglas \& McIntyre, Vancouver.

CARlson Keith Thor et Sonny McHalsie

1998, I am Stó:lō! Katherine explores her heritage, Stól:lō Heritage Trust, Chilliwack.

Collins June McCormick

1975, Valley of the spirits: upper Skagit Indians of Western Washington, University of Washington Press, Seattle.

DÉLÉAGE Pierre

2009, Le chant de l'anaconda, Société d'ethnologie, Nanterre.

Descola Philippe

2005, Par-delà nature et culture, Gallimard, Paris.

ELMENDORF William W.

1993, Twana narratives: native historical accounts of a Coast Salish culture, University of Washington Press/University of British Columbia Press, Seattle/Vancouver.

FRAZER James George

1887, Totemism, Adam \& Charles Black, Édimbourg.

Galloway Brent D.

1994, A grammar of upriver Halkomelem, University of California Press, Berkeley. 
GiLLe Baptiste

2014, « Modalités énonciatives du sacré chez les Salish centraux de la côte », Annales de la Fondation Fyssen, 28, p. 21-38.

GoldENweISER Alexander

1910, « Totemism: an analytical study », Journal of American folklore, 23, p. 17-293.

GuÉDON Marie-Françoise

1984, «An introduction to Tsimshian world-view and its practitionners », in Margaret Anderson (éd.), The Tsimshian: images of the past, views for the present, University of British Columbia Press, Vancouver.

HiLl-Tout Charles

1904, « Totemism: a consideration of its origin and import », Man, 4, p. 74-78.

1978, The Salish people: the local contribution of Charles Hill-Tout. Vol. 3: The mainland Halkomelem, Ralph Maud (éd.), Talcon books, Vancouver.

HultKRANTZ Åke

1953, The conception of the soul among North American Indians, Ethnographical Museum of Sweden, Stockholm.

JILEK Wolfgang G.

1974, Indian healing: shamanic ceremonialism in the Pacific Northwest today, Hancock House Publishers Ltd., Surrey.

Kew Michael

1970, Coast Salish ceremonial life: status and identity in a modern village, University of Washington Press, Washington.

LÉvi-Strauss Claude

1950, « Introduction à l'œuvre de Marcel Mauss », in Marcel Mauss, Sociologie et anthropologie, PUF, Paris.

1962a, Le totémisme aujourd'hui, PUF, Paris.

1962b, La pensée sauvage, Plon, Paris.

Miller Bruce Granville

2007, Be of good mind: essays on the Coast Salish, University of British Columbia Press, Vancouver.

MoHs Gordon

1994, « Stó:lō sacred grounds », in David L. Carmichael (éd.), Sacred sites, sacred slaces, Routledge, Londres/New York.

Neylan Susan

2011, " Shaking up christianity: the Indian Shaker church in the Canada-U.S. Pacific Northwest », The journal of religion, 91 (2), p. 188-222.

Robinson Sarah Anne

1963, Spirit dancing among the Salish Indians, Vancouver Island, British Columbia, University of Chicago, Chicago. 
Rosa Frederico

2003, L'âge d'or du totémisme : histoire d'un débat anthropologique (1887-1929), Éditions de la Maison des sciences de l'Homme, Paris.

RuBy Robert H. et John A. BRown

1996, John Slocum and the Indian Shaker church, University of Oklahoma Press, Norman.

SPERBER Dan et Deirdre WiLson

1989, La pertinence : communication et cognition, Les Éditions de Minuit, Paris.

Suttles Wayne P. et Ralph Maud

1987, Coast Salish essays, Talonbooks, Vancouver.

SutTles Wayne P. et Diamond JenNESS

1956, Katzie ethnographic notes/The faith of a Coast Salish Indian, Wilson Duff (éd.), British Columbia Provincial Museum, Victoria.

TURNER Nancy J.

2005, The earth's blanket: traditional teachings for sustainable living, University of Washington Press, Seatle.

VAN EIJIK Jan P.

2001, « Who is Sunutqaz? A Salish quest », Anthropological linguistics, 43 (2), p. 177-197.

WeLLs Oliver

1987, The Chilliwacks and their neighbors, Ralph Maud et Brent Douglas Galloway (éd.), Talonbooks, Vancouver.

WigGins David

1980, Sameness and substance, Harvard University Press, Cambridge.

WIKE Joyce Annabel

1941, Modern spirit dancing of Northern Puget Sound, University of Washington, Washington. 